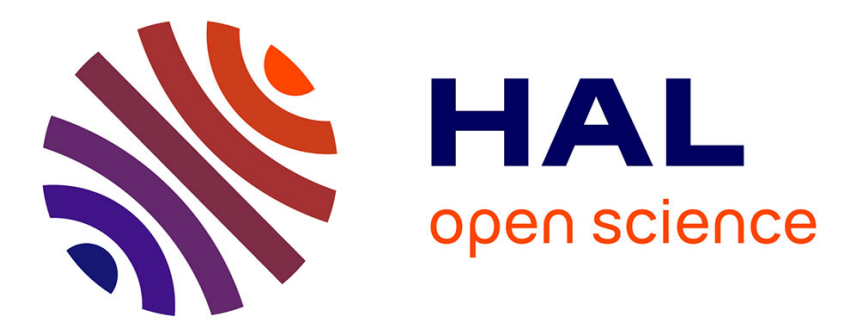

\title{
Contribution of passive actions to the lower limb joint moments and powers during gait: A comparison of models
}

Xavier Gasparutto, Eric Jacquelin, Raphaël Dumas

\section{- To cite this version:}

Xavier Gasparutto, Eric Jacquelin, Raphaël Dumas. Contribution of passive actions to the lower limb joint moments and powers during gait: A comparison of models. Proceedings of the Institution of Mechanical Engineers, Part H: Journal of Engineering in Medicine, 2018, 232 (8), pp.768-778. 10.1177/0954411918785661 . hal-01857851

\section{HAL Id: hal-01857851 \\ https://hal.science/hal-01857851}

Submitted on 17 Aug 2018

HAL is a multi-disciplinary open access archive for the deposit and dissemination of scientific research documents, whether they are published or not. The documents may come from teaching and research institutions in France or abroad, or from public or private research centers.
L'archive ouverte pluridisciplinaire HAL, est destinée au dépôt et à la diffusion de documents scientifiques de niveau recherche, publiés ou non, émanant des établissements d'enseignement et de recherche français ou étrangers, des laboratoires publics ou privés. 


\section{Contribution of passive actions to the lower limb joint moments and powers during gait: a comparison of models}

\begin{tabular}{|c|c|}
\hline Journal: & Part H: Journal of Engineering in Medicine \\
\hline Manuscript ID & JOEIM-17-0227.R1 \\
\hline Manuscript Type: & Original article \\
\hline Date Submitted by the Author: & $n / a$ \\
\hline Complete List of Authors: & $\begin{array}{l}\text { Gasparutto, Xavier; Univ Lyon, Université Claude Bernard Lyon 1, IFSTTAR, } \\
\text { LBMC UMR_T9406, F69622 } \\
\text { Jacquelin,Eric; Univ Lyon, Université Claude Bernard Lyon 1, IFSTTAR, } \\
\text { LBMC UMR_T9406, F69622 } \\
\text { Dumas, Raphael; Univ Lyon, Université Claude Bernard Lyon 1, IFSTTAR, } \\
\text { LBMC UMR_T9406, F69622 }\end{array}$ \\
\hline Keywords: & $\begin{array}{l}\text { Gait Analysis, Inverse Dynamics, Passive Moments, Ligament Model, Lower } \\
\text { Limb }\end{array}$ \\
\hline Abstract: & $\begin{array}{l}\text { The lower limb passive actions representing the actions of all the passive } \\
\text { periarticular structures have been shown to have a significant contribution } \\
\text { to the power generation and absorption during gait. However, the } \\
\text { respective magnitude of its different components was not established, } \\
\text { although models of ligament moment were implemented in some musculo- } \\
\text { skeletal models. These ligament moments have shown to have an influence } \\
\text { on the musculo-tendon forces and contact forces but the models used were } \\
\text { never specifically evaluated, i.e. compared to the passive and net joint } \\
\text { moments. Two models of passive joint moments and three models of } \\
\text { ligament moments were selected from the literature. Ten subjects ( } 23 \text { to } \\
29 \text { years old, } 79.8 \pm 9.5 \mathrm{~kg}, 1.85 \pm 0.06 \mathrm{~m} \text { ) participated in the study. Each } \\
\text { subject performed three gait cycles in a gait laboratory to acquire the } \\
\text { kinematics and ground reaction forces and to compute the ligament, } \\
\text { passive, and net moments of the right lower limb joints. The contributions } \\
\text { of passive joint moments to the net joint moments were in accordance with } \\
\text { the literature although time-shifts appeared for peaks in the hip and knee } \\
\text { powers. Two of the models of ligament moments seemed, in fact, to } \\
\text { represent the passive joint moments as their contributions were very } \\
\text { similar whilst the third model of ligament moments seemed to represent } \\
\text { only penalty-based joint limits. As a conclusion, this study showed that the } \\
\text { models of ligament moments existing in the literature do not seem reliable. } \\
\text { This study also demonstrated that the use of non subject-specific models of } \\
\text { the passive joint moments could be a valid approach for healthy subjects. }\end{array}$ \\
\hline
\end{tabular}


Contribution of passive actions to the lower limb joint moments and powers during gait: a comparison of models

\section{Xavier Gasparutto}

5 Univ Lyon, Université Claude Bernard Lyon 1, IFSTTAR, LBMC UMR_T9406, F69622, Lyon, France

e-mail: xavier.gasparutto@hotmail.fr

Eric Jacquelin

10 Univ Lyon, Université Claude Bernard Lyon 1, IFSTTAR, LBMC UMR_T9406, F69622, Lyon, France

e-mail: eric.jacquelin@univ-lyon1.fr

\section{Raphael Dumas}

15 Univ Lyon, Université Claude Bernard Lyon 1, IFSTTAR, LBMC UMR_T9406, F69622, Lyon, France

e-mail: raphael.dumas@ifsttar.fr 


\section{Keywords:}

20 Gait Analysis, Inverse Dynamics, Passive Moments, Ligament Model, Lower Limb

\section{Abstract}

The lower limb passive actions representing the actions of all the passive periarticular structures have been shown to have a significant contribution to the power generation and absorption during gait. However, the respective magnitude of its different components was not established, although models of ligament moment were implemented in some musculo-skeletal models. These ligament moments have shown to have an influence on the musculo-tendon forces and contact forces but the models used were never specifically evaluated, i.e. compared to the passive and net joint moments. Two models of passive joint moments and three models of ligament moments were selected from the literature. Ten subjects ( 23 to 29 years old, $79.8 \pm 9.5 \mathrm{~kg}, 1.85 \pm 0.06 \mathrm{~m}$ ) participated in the study. Each subject performed three gait cycles in a gait laboratory to acquire the kinematics and ground reaction forces and to compute the ligament, passive, and net moments of the right lower limb joints. The contributions of passive joint moments to the net joint moments were in accordance with the literature although time-shifts appeared for peaks in the hip and knee powers. Two of the models of ligament moments seemed, in fact, to represent the passive joint moments as their contributions were very similar whilst the third model of ligament moments seemed to represent only penalty-based joint limits. As a conclusion, this study showed that the models of ligament moments existing in the literature do not seem reliable. This study also demonstrated that the use of non subject-specific models of the passive joint moments could be a valid approach for healthy subjects. 


\section{List of Abbreviations}

\begin{tabular}{|c|c|c|}
\hline Models & $\mathrm{P}^{1}$ & First Passive Joint Moment Model \\
\hline & $\mathrm{P}^{2}$ & Second Passive Joint Moment Model \\
\hline & $\mathrm{L}^{1}$ & First Ligament Moment Model \\
\hline & $\mathrm{L}^{2}$ & Second Ligament Moment Model \\
\hline & $\mathrm{L}^{3}$ & Third Ligament Moment Model \\
\hline
\end{tabular}

Angles FE Flexion - Extension

AA Adduction - Abduction

IER Internal - External Rotation

Peaks $\quad \mathrm{HM}^{\mathrm{i}} \quad \mathrm{i}^{\text {th }}$ Peak Moment at the Hip

$\mathrm{HP}^{\mathrm{i}} \quad \mathrm{i}^{\text {th }}$ Peak Power at the Hip

$\mathrm{KM}^{\mathrm{i}} \quad \mathrm{i}^{\text {th }}$ Peak Moment at the Knee

$\mathrm{KP}^{\mathrm{i}} \quad \mathrm{i}^{\text {th }}$ Peak Power at the Knee

$\mathrm{AM}^{\mathrm{i}} \quad \mathrm{i}^{\text {th }}$ Peak Moment at the Ankle

$\mathrm{AP}^{\mathrm{i}} \quad \mathrm{i}^{\text {th }}$ Peak Power at the Ankle

Misc ISB International Society of Biomechanics

JCS Joint Coordinate System

\section{Introduction}

The assessment of the contributions of the joint passive actions to the net joint moment and power can help understanding the underlying mechanisms of locomotion. The passive actions at the hip, knee and ankle joints have been shown to have a significant contribution to the power generation and absorption during gait [1-5]. These passive

55 joint actions represent the actions of all of the passive structures crossing the joint, including the passive components of the muscle-tendon complex and ligaments. However, the respective contribution of these components within the passive joint moments was not established although models of ligaments moments were implemented in some musculo-skeletal models [6-8]. When taken into account in musculoskeletal

60 models, the ligament moments have shown to have a moderate influence on the muscle forces estimation and to tend to reduce the joint reaction forces during gait [9]. 
To understand the contribution of the ligaments to the passive moments, we propose to implement, within an inverse dynamics framework, the models of the ligament moments that were used in musculo-skeletal modelling $[6,8,10,11]$ and to compare

65 them to the passive and net joint moments. The net joint moments represent the moments applied by the proximal segment on the distal segment at a point taken as the joint centre and include all mechanical actions of the periarticular structures, both active and passive. The models of passive joint moment give an estimate of the moment resulting from the actions of the passive structures around one joint axis as a function of 70 the joint angles. The ligament moment models are similar but give an estimate of the moment resulting from the ligament action. Thus, the ligament moments are a component of the passive joint moments which are themselves a component of the net joint moments. This definition will allow us to assess qualitatively the reliability of the different models with the following assumptions: the modelled ligament moment should

75 be lower than the modelled passive joint moments and the modelled passive joint moment should be lower than the net joint moment computed by an inverse dynamics method. Protocols to define patient specific models of passive joint moments have been defined $[2,12]$. However, the procedure can be costly in a clinical environment, therefore, we preferred to select and assess generic models from the literature. Two 80 generic models of passive joint moments $[13,14]$ and three models of ligament moments $[6,8,10,11]$ were adapted to the joint coordinate systems (JCS) standard of the International Society of Biomechanics (ISB) $[15,16]$. The ligament, passive, and net joint moments were evaluated through an inverse dynamics analysis on ten healthy male subjects. The contribution of the passive joint moments to the net joint moments were compared to previous results [2] obtained with subject specific models.

We hypothesize that the contribution of the ligament moments to the passive joint 
moments will be negligible apart when the joints reach the end of their range of motion.

\section{Materials \& Methods}

\section{Measurements}

90 This study was retrospective and used data previously published [17-19]. The measurements were done under the Helsinki accord and every subjects gave an informed consent. Ten healthy male subjects $(26 \pm 2.4$ years, $73.5 \pm 15 \mathrm{~kg}, 1.75 \pm 0.06 \mathrm{~m})$ performed 3 gait cycles each at their self-selected speed. Their right lower limb was analysed. The gait cycle was defined from heel contact to the next heel contact of the

95 right foot. The subjects were equipped with 14 reflective markers taped on anatomical landmarks of the right lower limb and pelvis (Right/Left Anterior Superior Iliac Spines, Right/Left Posterior Superior Iliac Spines, Greater Trochanter, Medial/Lateral Femoral Epicondyle, Tibial Tuberosity Advancement, Fibula Head, Medial/Lateral Malleoli, Calcaneous, $1^{\text {st }}$ and $5^{\text {th }}$ Metatarsal Heads). The trajectories of the skin markers were 100 recorded at $100 \mathrm{~Hz}$ by 8 Eagle ${ }^{\circledR}$ cameras (Motion Analysis Corporation, Santa Rosa, CA, USA) and the ground reaction forces and moments were recorded by two force plates (Bertec Corporation, Columbus, OH, USA) embedded in the walkway. These measurements were used to acquire the kinematics of the right lower limb joints during gait and to compute the net joint moments and powers.

\section{Models of passive joint moment and ligament moment}

\section{Passive joint moment}

The generic models of passive joint moment were chosen on their capacity to model some specifications due to the characteristics of the passive structures described in the literature $[5,10,11,13,20-26]$ : taking into account bi-articular muscles, having a 
110 pattern in double exponentials, being based on in vivo measurements and, to have all the parameters of the model available. To the authors' knowledge, the model of Riener et al. [13] and the model of Amankwah et al. [14] are the only one fitting these specifications.

Model $P^{l}$ : The parameters of the first model of passive joint moment $\left(P^{1}\right)$ were

115 identified by Riener et al. in a previous study [13] on 10 subjects (23 to 29 years old, $79.8 \pm 9.5 \mathrm{~kg}, 1.85 \pm 0.06 \mathrm{~m})$. This model describes the passive flexion-extension (FE) moment of the three main joints of the lower limb (hip, knee, and ankle, i.e., both tibiotalar and subtalar joints). It needed slight adaptation (i.e., axes permutation) to fit the standard of the ISB $[15,16]$. Indeed, the FE axis directions were different from the ISB

120 standards. The adjusted model behaviour is depicted in Figure 1. The curves of the passive ankle, knee, and hip moments were exponentials depending on two (or three) of the corresponding joint angles. The equation to obtain the amplitude of the passive joint moment is written below (Eqs. (1) and (2)) and the adjusted parameters can be found in Table 1.

$$
P_{i}^{1}=e^{\left(\alpha_{i}+h_{i}^{1} \cdot \theta_{H}+k_{i}^{1} \cdot \theta_{K}+a_{i}^{1} \cdot \theta_{A}\right)}-e^{\left(\beta_{i}+h_{i}^{2} \cdot \theta_{H}+k_{i}^{2} \cdot \theta_{K}+a_{i}^{2} \cdot \theta_{A}\right)}+C_{i}
$$

where $\theta_{i}$ is the FE angle of the joint ( $i=H$ for hip, $K$ for knee, and A for ankle). Parameters $h_{i}^{j}, k_{i}^{j}$ and $a_{i}^{j}(j=1,2)$ are related to the bi-articular muscle passive actions, $\alpha_{i}$ and $\beta_{i}$ are scaling factors and $C_{i}$ is a constant. The corresponding hip, knee, and ankle FE axes are presented in the section Joint angles and moments axes.

130 At the knee, a third term $p_{K}^{*}$ needs to be added to $P_{K}$ to have the final total passive moment:

$$
p_{K}^{*}=-e^{\left(2,22+0,15 \cdot \theta_{K}\right)}
$$


Model $P^{2}$ : The parameters of the second model of passive joint moment $\left(P^{2}\right)$ were identified by Amankwah et al. in a previous study [14] on 5 able-bodied subjects ( 2 135 females and 3 males, 23 to 29 years old, $67.7 \pm 13.3 \mathrm{~kg}, 1.74 \pm 0.16 \mathrm{~m})$. This model describes the viscoelastic passive moments of the hip in FE and adduction-abduction (AA), of the knee in FE and of the ankle in FE and inversion-eversion. As the present paper focus on the elastic passive joint moment, only the exponential elastic components of this model was used.

140 The parameters sign of the model have been adjusted (i.e., axes permutation) to match the ISB standards $[15,16]$. The adjusted model is depicted in Figure 2. As for model $P^{l}$, the curves of the passive ankle, knee, and hip moments were exponentials depending on two (or three) of the corresponding joint angles. The model equation is presented in equation (3) and the adjusted parameters in Table 2:

$$
P_{i}^{2}=a_{1} \cdot e^{\left(a_{2} \cdot \theta+a_{3} \cdot \theta_{p}+a_{4} \cdot \theta_{d}\right) \cdot \pi / 180}+a_{5} \cdot e^{\left(a_{6} \cdot \theta+a_{7} \cdot \theta_{p}+a_{8} \cdot \theta_{d}\right) \cdot \pi / 180}
$$

where $a_{1 \rightarrow 8}$ are the model parameter, $\theta$ is the angle of the considered joint, $\quad \theta_{p}$ is the angle of the proximal joint and $\theta_{d}$ is the angle of the distal joint. The angles are defined in degree and the proximal and distal joint for each degree of freedom are defined in Table 2. The corresponding hip, knee, and ankle axes are presented the section Joint angles and moments axes.

\section{Ligament moment}

Only three models $[8,10,11]$ that describe the action of the ligaments at the three main joints of the lower limb were found in the literature. These models were used in musculoskeletal models $[6-8]$ and described $2 \mathrm{D}[10,11]$ or $3 \mathrm{D}$ actions $[6,8]$ of the

155 ligaments. They were adapted in the present study to match the ISB standards and to 
present patterns consistent with the functional anatomy of the joints [27]. The patterns of the ligament moments as a function of the joint angles were presented in Figure 3. For the three models, the curves of the ligament ankle, knee, and hip moments were exponentials (or lines) depending only on the corresponding joint angle.

160 Linear model: The passive joint moments, representing either the ligament or all periarticular structures, are known to behave as double exponential $[11,13]$. However, Al Nazer et al. [6] presented a linear model of ligament moment. It might seem irrelevant with regard to the physiological behaviour of those structure. Nevertheless, it was tested in the present study to assess the validity of a linear model for the estimation 165 of ligament actions. This model was based on in-vivo measurements [14]. It was adjusted (i.e., axes permutation) in the present study to fit the ISB standards $[15,16]$ and a neutral angle $\theta_{0}$ (where the ligament moment is null) was added. Its value was chosen to be consistent with the functional anatomy of the joint [27] and the general patterns of passive actions $[5,10,11,13,21-23,25,26]$. The equation to obtain the

170 amplitude of the ligament moment $L^{1}$ is written below (4). The linear model considered three joint angles at the hip, namely FE, AA, and internal-external rotation (IER) and only the FE angle at the knee and ankle. The moment axes are presented in the section Joint angles and moments axes.

$$
L_{i}^{1}=-k_{i}\left(\theta_{i}-\theta_{i}^{0}\right)
$$

175 where $i$ indicates the joint ( $i=H$ for hip, $K$ for knee, and $A$ for ankle), $k_{i}$ is the passive stiffness in $\mathrm{Nm} / \mathrm{deg}, \theta_{i}$ is the joint angle in degree and $\theta_{i}^{0}$ is the neutral joint angle in degree. The adjusted parameters can be found in Table 2 .

Exponential models: Two exponential models were considered and adjusted (i.e., axes permutation) to the ISB standards. A first double exponential model $\left(L^{2}\right)$ was adjusted in 
180 the present study from two papers of Audu and Davy $[10,11]$ based on the in vivo measurements of Hatze [28]. The hip and knee models were based on their 1985 study [11] and the ankle model was based on their 1987 study [10]. The general equation to obtain the amplitude of the ligament moment is written below (5). The moment axes are presented in the section Joint angles and moments axes.

$$
L_{i}^{2}=c_{i} e^{-a_{i}\left(\theta_{i}-\alpha_{i}\right)}+d_{i} e^{-b_{i}\left(\beta_{i}-\theta_{i}\right)}
$$

where $i$ represent the joint ( $i=H$ for hip, $K$ for knee, and $A$ for ankle), $c_{i}$ and $d_{i}$ are scaling parameters in $\mathrm{Nm}$ that manage the magnitude of the moment, $a_{i}$ and $b_{i}$ are parameters linked to the stiffness, the higher they are, the higher the stiffness of the joint will be, $\theta_{i}$ is the joint angle in degree and $\left[\alpha_{i}, \beta_{i}\right]$ is the neutral zone of the angular range of motion of the joint, where the passive moments are minimal. The parameters adjusted to the ISB standards can be found in Table 3.

The second double exponential model $\left(L^{3}\right)$ [8] was developed by Anderson et al. for forward dynamic simulations. The formulation was similar to the formulation of $L^{2}$. Some adjustments had to be made in the present study: the angular range of the neutral 195 zone at the knee and ankle were too wide in the initial model when compared to literature $[10,11,29]$. As an example, the knee ligament moment started increasing at 7.5deg of extension, an angle rarely seen for healthy subjects. Thus the neutral zone of the knee and ankle $L^{3}$ were modified to match, respectively, the neutral zone of the model $L^{2}$ and of the simulation study by Imhauser et al. [29]. The general equation to 200 obtain the amplitude of the ligament moment is written below (6). The moment axes are presented in the section Joint angles and moments axes.

$$
L_{i}^{3}=c_{i} e^{a_{i}\left(\theta_{i}-\alpha_{i}\right)}+d_{i} e^{b_{i}\left(\theta_{i}-\beta_{i}\right)}
$$

The parameter definitions are the same as for $L_{i}^{2}$ even if the formulation is slightly 
different. The adjusted parameters can be found in Table 4 .

As the amplitude of the passive joint moments and ligament moments are function of the joint angles, it seemed appropriate to express both the moments and angles according to the joint coordinate system (JCS). Therefore, the joint angles were expressed about the axes $\mathbf{e}_{1}, \mathbf{e}_{2}, \mathbf{e}_{3}$ of the JCS while the passive joint moments and

210 ligament moments were expressed about the dual axes $\mathbf{e}^{1}, \mathbf{e}^{2}, \mathbf{e}^{3}$ defined in (7) [30, 31]:

$$
\left\{\begin{array}{l}
\mathbf{e}^{1}=\frac{\mathbf{e}_{2} \times \mathbf{e}_{3}}{\left(\mathbf{e}_{1} \times \mathbf{e}_{2}\right) \mathbf{e}_{3}} \text { and } \mathbf{e}^{1} \sqsubset \mathbf{e}_{1}=1 \\
\mathbf{e}^{2}=\mathbf{e}_{2} \\
\mathbf{e}^{3}=\frac{\mathbf{e}_{1} \times \mathbf{e}_{2}}{\left(\mathbf{e}_{1} \times \mathbf{e}_{2}\right) \mathbf{e}_{3}} \text { and } \mathbf{e}^{3} \sqsubset \mathbf{e}_{3}=1
\end{array}\right.
$$

As a consequence, the joint angular velocity, for instance for the hip, was the vector

$$
\boldsymbol{\omega}_{H}=\dot{\theta}_{H, 1} \mathbf{e}_{H, 1}+\dot{\theta}_{H, 2} \mathbf{e}_{2}+\dot{\theta}_{H, 3} \mathbf{e}_{H, 3} .
$$

with $\dot{\theta}_{H, 1}, \dot{\theta}_{H, 2}$ and $\dot{\theta}_{H, 3}$ the time derivative of the FE angle, AA angle and IER angle.

215 For the passive joint moments and ligament moments, this means that the FE passive moment amplitude was not applied on the FE axis $\mathbf{e}_{1}$ but on the axis $\mathbf{e}^{1}$ (i.e., orthogonal to both $\mathbf{e}_{2}$ and $\mathbf{e}_{3}$ ). This applied as well for the other degrees of freedom, leading, as an example, to the following expression for the linear ligament moment at the hip (9) and the exponential ligament moment at the knee (10):

$$
\begin{gathered}
\mathbf{L}_{H}^{1}=-\left(k_{H, 1}\left(\theta_{H, 1}-\theta_{H, 1}^{0}\right) \mathbf{e}_{H}^{1}+k_{H, 2}\left(\theta_{H, 2}-\theta_{H, 2}^{0}\right) \mathbf{e}_{H}^{2}+k_{H, 3}\left(\theta_{H, 3}-\theta_{H, 3}^{0}\right) \mathbf{e}_{H}^{3}\right) \\
\mathbf{L}_{K}^{2}=\left(c_{K} e^{-a_{K}\left(\theta_{K}-\alpha_{K}\right)}+d_{K} e^{-b_{K}\left(\beta_{K}-\theta_{K}\right)}\right) \mathbf{e}_{K}^{1}
\end{gathered}
$$

Note that, $\mathbf{e}_{i} \mathbf{e}^{j}=\delta_{i}^{j}$ (i.e., Kronecker symbol) [30]. This property was used for the computation of the powers corresponding to the passive joint moments and ligament 
moments, i.e., the dot products $\mathbf{P} \square \boldsymbol{\omega} \quad$ and $\mathbf{L} \square \boldsymbol{\omega}$.

\section{Inverse dynamics}

Inverse dynamics was performed by using natural coordinates [19] to compute the net joint moments at the main three joints of the lower limb: hip, knee and ankle. The net moment at a joint was defined as the moment applied by the proximal segment on the distal segment computed at a point defined as the proximal joint centre. The

230 components of net joint moment in the JCS were computed by projection (i.e. dot product with the axes $\mathbf{e}_{1}, \mathbf{e}_{2}, \mathbf{e}_{3}$ ). The passive moments and ligament moments were computed based on the lower limb kinematics, separately from the inverse dynamics.

The joint powers were computed as the dot products of the moments and the joint angular velocities (e.g. angular velocities of the distal segments with respect to the 235 proximal segments).

Dimensionless moments and powers were then computed [32] to compare moments and powers between subjects. Moments and powers were respectively divided by $\eta_{M}$ and $\eta_{P}$ defined in (11):

$$
\left\{\begin{array}{l}
\eta_{M}=m \cdot g \cdot \frac{T}{2} \\
\eta_{P}=m \cdot\left(g^{3} \cdot \frac{T}{2}\right)^{1 / 2}
\end{array}\right.
$$

240 where $m$ is the mass of the subject in $\mathrm{kg}, g$ the acceleration of gravity (meters.seconds ${ }^{-2}$ ) and $T$ the height of the subject in meters. The height was divided by two to have an estimation of the length of the lower limb of the subject.

\section{Results}

The joint angles, net joint moments and powers, passive joint moments and powers, and 
245 ligaments moments and powers at the hip, knee and ankle are presented in Figure 4 to 7. The main contributions of the passive joint moments and powers, and of the ligament moments and powers to the net joint moments and powers are reported in Table 6 . The contributions obtained by Whittington et al. [2] are given for comparison. A negative contribution represents an action opposite to the net joint moment or power.

250 The main contributions of $P^{1}$ were at the hip, knee and ankle during the single support phase and the push off phase and at the knee during the late swing. Time-shifts were observed between the net peaks and the corresponding $P^{1}$ peaks at $H P^{3}$ (Fig. 4) and $K P^{5}$ (Fig. 6). At the ankle, during the swing phase, a passive joint moment peak $\left(A M^{2}\right)$ and two peaks of passive joint power $\left(A P^{4}, A P^{5}\right)$ were observed without any corresponding 255 net joint moment or power.

The main contributions of $P^{2}$ at the hip were during the double support and push-off phase. A peak extension moment accounting for $80 \%$ of $H M^{3}$ FE was observed with a time-shift of $20 \%$ responsible for a resistive power peak during the swing phase. The hip passive AA joint moment of $P^{2}$ had a similar pattern as the ligament moment $L^{1}$ but

260 with an offset resulting in an adduction moment during the whole gait cycle. The main contributions to the knee net joint moment were during the single support phase and late swing. At the ankle, the contribution to the net joint moment and power was below $10 \%$ during the push-off phase and above $10 \%$ during the opposite toe-off phase.

The main contributions of $L^{1}$ were at the hip and knee during the double support phase, 265 the single support phase, the push off, the toe-off and the late swing. During the main part of the swing phase, the moment and power generated by $L^{1}$ were contrary to the FE net joint moment and power at the knee but with similar patterns. Time-shifts were observed between the net peaks and the corresponding $L^{1}$ peaks at $H P^{3}$ (Fig. 2) and $K P^{5}$ 
(Fig 6). At the ankle, the contributions were below 10\% except for the net joint power

270 during the opposite toe-off. During the swing phase, a peak ligament moment $\left(A M^{2}\right)$ and peak ligament power $\left(A P^{4}\right)$ were observed without any corresponding net joint moment or power.

The main contributions of $L^{2}$ were at the hip and knee during the single support phase, the push off, the toe-off, and the late swing. The contribution to the net joint moment 275 and power at the ankle were below 10\%. Time-shifts were observed between the net peaks and the corresponding $L^{2}$ peaks at $H P^{3}$ (Fig. 4). At the ankle, during the swing phase, a peak ligament moment $\left(A M^{2}\right)$ was observed without any corresponding net joint moment.

$L^{3}$ had no substantial contribution.

\section{Discussion}

This study compared the models of ligament moments used in musculoskeletal modelling to passive and net moments. To that end, five existing models, two representing the actions of all anatomical structures spanning the ankle, knee, and hip joints and three representing only the actions of the ligaments, were adjusted to fit the

285 ISB standards for the joint axes definition and, for some models, to fit the neutral range (i.e. range of minimal passive moments) described in functional anatomy. The main limitation of this study was the use of generic models instead of subject specific models as in Whittington et al. [2]. This will be discussed hereafter, especially regarding the validity of using generic models to estimate the passive joint moments for healthy 290 subjects. 


\section{Passive joint moment}

To the author's knowledge, the selected generic passive joint moments were never assessed during gait. Although time-shifts were observed for several peaks $\left(\mathrm{HP}^{3}\right.$ and $\mathrm{KP}^{5}$ ), the results obtained with the passive joint models $P^{1}$ and $P^{2}$ were consistent with

295 the literature that showed a substantial role of the passive periarticular structure during gait [1-5] at the hip, knee and ankle. The results with model $P^{1}$ were closer to the results of Whittington et al. [2] than the results with the model $P^{2}$ at both the hip and ankle (Table 6). For instance, the contributions at peak $H M_{\mathrm{FE}}^{3}$ were $0 \%$ with model $P^{1}$ and in [2] but were $80 \%$ with model $P^{2}$. The contributions at peak $A M^{1}$ were $20 \%$ and $19 \%$ 300 with model $P^{1}$ and in [2] and were $6 \%$ with model $P^{2}$. This could be explained by the fact that the subjects of the present study were in the same range of age, size, and weight and had the same sex as the population of Riener and Edrich [13] used to establish model $P^{1}$. Moreover, the model $P^{2}$ was based on 5 subjects with large anthropometric variation where model $P^{1}$ was based on 10 subjects with smaller

305 anthropometric variations. Nevertheless, the differences between the present results and the literature as well as the time-shifts could be attributed to the different methods used to compute the net joint moments and powers [33].

At the hip, to the authors' knowledge, the AA passive joint moment during gait was not evaluated previously. The model $P^{2}$ presented a similar pattern to $L^{1}$ with an offset

310 leading to a passive adduction moment during the whole gait cycle. However, this result should be taken with care as Amankwah et al. [14] stated that the hip model had a poor fit with their experimental data.

\section{Generic models}


The results of the joint passive moments taken from the literature show the 315 practicality of using a generic model when studying non-pathological subjects. The model from Riener and Edrich [13] seems to give consistent results and has been used as a reference for validation of musculoskeletal models in previous studies $[35,36]$. Consistently, the contribution of the muscle-generated passive joint moments to the total (i.e., both passive and active) muscle-generated joint moments at the hip, knee and

320 ankle during gait, as reported by Rajagopal et al., [36], revealed patterns very similar to the results obtained in the present study with model $P^{1}$.

It might be necessary to define models for different groups defined by age, sex, weight or size as the model $P^{2}$ showed lower contributions at the ankle and knee. However, the few studies that looked at the influence of age or sex on passive joint 325 moments $[1,34]$ showed no significant differences across groups for non-pathological subjects.

\section{Ligament moment}

Concerning the ligament moments, the models $L^{1}$ and $L^{2}$ seemed to overestimate the actions of the ligaments as, most of the time, those actions were comparable or superior to the passive joint moment and power at $H P^{2}$ and $H P^{3}$ for $L^{1}$ and at $K M^{2}$ and $K M^{3}$ for $L^{2}$. As the passive joint moment regroups the actions of all the passive components of the joint including the inactive muscles and the ligaments, it is very unlikely that the magnitude of the moment generated by the ligaments only would be equal or superior to that moment. This issue might come from a confusion made by the authors in the

335 definition of the models. Indeed, for the model $L^{1}$, Al Nazer et al. [6] stated that his model was based on experimentations and cited a study measuring the passive joint moments [14] although the model is presented and used in their musculoskeletal model 
as a ligament model. The model $L^{2}[10]$ was also presented as a model of ligament actions and used in a musculoskeletal model [37]. However, the authors only modified 340 the neutral range of their model developed in Audu and Davy [11] without modifying the magnitude and added new parameters for the ankle model without explaining their origin. In addition, the model from Audu and Davy [11] was presented as a ligament model but was based on a study by Hatze [28] that took into account all the periarticular structures. The fact that that Piazza and Delp [37] scaled $L^{2}$ at the hip to $75 \%$ of the 345 value reported by Audu and Davy [11] also indicates that this model might overestimate the ligament actions. Therefore, it looks like $L^{1}$ and $L^{2}$ are taking into account all passive actions and not only the ligament actions. This could lead to a redundancy of passive actions when these models were implemented in musculoskeletal models such as in Dumas et al. [9] for $L^{1}$ and in Piazza and Delp [37] at the hip and knee joints for $350 L^{2}$.

Assuming that the linear model $L^{1}$ in fact represented the passive actions of the whole periarticular structures, it was able to replicate the pattern of $P^{1}$ and $P^{2}$ but seemed to overestimate passive actions for high extension at the hip and high flexion at the knee. Therefore, as expected, this linear model of passive actions does not seem adapted to 355 describe the behaviour of the passive structures at the hip and knee. Nevertheless, at the ankle, the amplitude and pattern of $L^{1}$ and $P^{2}$ were similar and $L^{1}$ had the same pattern as $P^{1}$ with a lower amplitude. Therefore, a linear model could eventually be adequate to describe the ankle passive actions.

The model $L^{3}$ did not have a major influence except for the late swing at the knee and 360 for the net joint moment of extension at the ankle. However, these net joint moments led to negligible powers. Thus, as this model was developed for forward dynamic 
musculoskeletal simulations, it can be assumed that its purpose was mainly to limit the joint range of motion and not to describe the physiological behaviour of the ligaments. This was confirmed by its pattern in Fig. 3 as it is null on the whole neutral range of FE 365 of a healthy knee and shows a very steep increase of ligament moments near the joint limits. Moreover, no information was available on the origin of the model parameters. Thus, this model did not seem adapted to describe the actions of the ligaments. This was somewhat contradictory with the statement reported by Correa et al. that the model $L^{3}$ "included the effects of ligament action at the hip, knee, and ankle joints" [38].

370 From one model of ligament moment to another, the behaviour varied a lot, from an observed overestimation of the actions to an absence of action. Therefore, it seems that a simple and reliable model of ligament moment that could be implemented in musculoskeletal models is not presently available in the literature. Such models could be defined by identifying the resultant action of the ligament with computational models

375 including the ligament properties [39-41] as it was done at the ankle by Imhauser et al. [29]. A second method could be through ex-vivo measurements [42]. Further study is needed to assess the passive actions of the ligament and articular capsule during gait.

As a conclusion, the present study is the first to assess the models of ligament moment used in musculoskeletal modelling by comparing them to passive and net joint 380 moments. The models of ligament moment currently available in the literature were not found reliable, and thus, no conclusion could be drawn on their contributions to the net joint moments and powers. Further study should look specifically into the passive actions of the ligaments, especially at the knee, to get a better understanding of their contributions. Regarding the joint passive moments models, although time-shifts 385 between peaks could be observed, the generic models showed contributions to the net 
moments that were previously observed with personalized models. This suggest the validity of the generic models approach for the estimation of joint passive moments in the lower limb of healthy subjects.

\section{References}

390 [1] Silder A, Heiderscheit B, Thelen DG. Active and passive contributions to joint kinetics during walking in older adults. J Biomech 2008; 41: 1520-1527.

[2] Whittington B, Silder A, Heiderscheit B, et al. The contribution of passive-elastic mechanisms to lower extremity joint kinetics during human walking. Gait Posture 2008; 27: 628-634.

[3] Mansour J, Audu M. The passive elastic moment at the knee and its influence on human gait. J

395

[4] Sasaki K, Neptune RR, Kautz S a. The relationships between muscle, external, internal and joint mechanical work during normal walking. J Exp Biol 2009; 212: 738-744.

[5] Yoon YS, Mansour JM. The passive elastic moment at the hip. J Biomech 1982; 15: 905-10.

[6] Al Nazer R, Rantalainen T, Heinonen a, et al. Flexible multibody simulation approach in the analysis of tibial strain during walking. J Biomech 2008; 41: 1036-1043.

[7] Neptune RR, Sasaki K, Kautz $\mathrm{S}$ a. The effect of walking speed on muscle function and mechanical energetics. Gait Posture 2008; 28: 135-143.

[8] Anderson F, Pandy M. A dynamic optimization solution for vertical jumping in three dimensions. Comput Methods Biomech Biomed Engin 1999; 2: 201-31.

405 [9] Dumas R, Moissenet F, Gasparutto X, et al. Influence of joint models on lower-limb musculotendon forces and three-dimensional joint reaction forces during gait. Proc Inst Mech Eng Part $H$ J Eng Med 2012; 226: 146-160.

[10] Davy D, Audu M. A dynamic optimization technique for predicting muscle forces in the swing phase of gait. J Biomech 1987; 20: 187-201.

410 [11] Audu ML, Davy DT. The influence of muscle model complexity in musculoskeletal motion modeling. J Biomech Eng 1985; 107: 147-157.

[12] Silder A, Whittington B, Heiderscheit B, et al. Identification of passive elastic joint moment-angle relationships in the lower extremity. J Biomech 2007; 40: 2628-2635.

[13] Riener R, Edrich T. Identification of passive elastic joint moments in the lower extremities. J Biomech 1999; 32: 539-44.

[14] Amankwah K, Triolo RJ, Kirsch R. Effects of spinal cord injury on lower-limb passive joint moments revealed through a nonlinear viscoelastic model. J Rehabil Res Dev 2004; 41: 15-32.

[15] Wu G, Siegler S, Allard P, et al. ISB recommendation on definitions of joint coordinate system of various joints for the reporting of human joint motion-part I: ankle, hip, and spine. $J$ Biomech 2002; 35: 543-548.

[16] Wu G, Cavanagh PR. ISB Recommendations in the Reporting for Standardization of Kinematic Data. J Biomech 1995; 28: 1257-1261.

[17] Duprey S, Cheze L, Dumas R. Influence of joint constraints on lower limb kinematics estimation from skin markers using global optimization. J Biomech 2010; 43: 2858-2862.

425 [18] Dumas R, Cheze L. Hip and knee joints are more stabilized than driven during the stance phase of gait : An analysis of the 3D angle between joint moment and joint angular velocity. Gait Posture 2008; 28: 243-250.

[19] Dumas R, Chèze L. 3D inverse dynamics in non-orthonormal segment coordinate system. Med 
Biol Eng Comput 2007; 45: 315-322.

430 [20] Lee RY, Munn J. Passive moment about the hip in straight leg raising. Clin Biomech (Bristol, Avon) 2000; 15: 330-4.

[21] Silder A, Whittington B, Heiderscheit B, et al. Identification of passive elastic joint moment-angle relationships in the lower extremity. J Biomech 2007; 40: 2628-35.

[22] Vrahas MS, Brand RA, Brown TD, et al. Contribution of passive tissues to the intersegmental moments at the hip. J Biomech 1990; 23: 357-362.

[23] McFaull SR, Lamontagne M. In vivo measurement of the passive viscoelastic properties of the human knee joint. Hum Mov Sci 1998; 17: 139-165.

[24] Piziali RL, Rastegar JC. Measurement of the nonlinear, coupled stiffness characteristics of the human knee. J Biomech 1977; 10: 45-51.

440 [25] Hajrasouliha a R, Tavakoli S, Esteki a, et al. Abnormal viscoelastic behaviour of passive ankle joint movement in diabetic patients: an early or a late complication? Diabetologia 2005; 48: 1225-8.

[26] Heerkens $Y$, Woittiez R. Inter-individual differences in the passive resistance of the human knee. Hum Mov Sci 1985; 4: 167-188.

445 [27] Kapandji IA. The physiology of the joints: Lower limb, Volume 2, 6th revised edition. Book, Churchill Livingstone, 2010.

[28] Hatze H. The complete optimization of a human motion. Math Biosci 1976; 135: 99-135.

[29] Imhauser CW, Siegler S, Udupa JK, et al. Subject-specific models of the hindfoot reveal a relationship between morphology and passive mechanical properties. J Biomech 2008; 41 : 1341-1349.

[30] O'Reilly O, Sena M, Feeley B, et al. On representations for joint moments using a joint coordinate system. J Biomech Eng 2013; 135: 114504.

[31] Dumas R, Cheze L. Letter to the Editor: Joint Moments in the Joint Coordinate System, Euler or Dual Euler Basis. J Biomech Eng 2014; 136: 4-5.

455 [32] Hof L. Scaling gait data to body size. Gait Posture 1996; 4: 222-3.

[33] Dumas R, Nicol E, Chèze L. Influence of 3D Inverse Dynamics Method on the Joint Forces and Moments During Gait. J Biomed 2007; 129: 786-90.

[34] Anderson DE, Madigan ML, Nussbaum M a. Maximum voluntary joint torque as a function of joint angle and angular velocity: model development and application to the lower limb. $J$ Biomech 2007; 40: 3105-13.

[35] Arnold EM, Ward SR, Lieber RL, et al. A model of the lower limb for analysis of human movement. Ann Biomed Eng 2010; 38: 269-279.

[36] Rajagopal A, Dembia C, DeMers M, et al. Full body musculoskeletal model for muscle-driven simulation of human gait. IEEE Trans Biomed Eng; in press. Epub ahead of print 2016. DOI: 10.1109/TBME.2016.2586891.

[37] Piazza S, Delp S. The influence of muscles on knee flexion during the swing phase of gait. J Biomech 1996; 29: 723-733.

[38] Correa TA, Pandy MG, Crossley KM. Response to comment on 'Contributions of individual muscles to hip joint contact force in normal walking [J. Biomech. 43 (2010) 1618-1622]'. J $470 \quad$ Biomech 2010; 43: 3070-3071.

[39] Smith CR, Lenhart RL, Kaiser J, et al. Influence of Ligament Properties on Tibiofemoral Mechanics in Walking. J Knee Surg 2015; 29: 99-106.

[40] Razu SS, Guess TM. EMG-driven Forward Dynamics Simulation to Estimate in Vivo Joint Contact Forces During Normal, Smooth, and Bouncy Gait. J Biomech Eng. Epub ahead of print 2017. DOI: 10.1115/1.4038507.

[41] Marouane H, Shirazi-Adl A, Adouni M. Knee joint passive stiffness and moment in sagittal and 
frontal planes markedly increase with compression. Comput Methods Biomech Biomed Engin 2015; 18: 339-350.

[42] Lamberto G, Richard V, Dumas R, et al. Modeling the human tibio-femoral joint using ex vivo determined compliance matrices. J Biomech Eng; 138.

[43] Shelburne KB, Torry MR, Pandy MG. Contributions of muscles, ligaments, and the groundreaction force to tibiofemoral joint loading during normal gait. J Orthop Res 2006; 24: 1983-90. 


\section{Figure Caption:}

Figure 1: Passive joint moments of model L1 in function of joint angles on typical gait joint range of motion, the colour variation from red to yellow indicates the angle variation between the extreme values defined in the legend of the graph.

490 Figure 2: Passive joint moments of model L2 in function of joint angles on typical gait joint range of motion, the colour variation from red to yellow indicates the angle variation between the extreme values defined in the legend of the graph.

Figure 3: Ligaments moments of models L1, L2 and L3 in function of joint flexion Figure 4: Hip flexion(+)/extension(-) (FE) - Kinematics, moment and power, net vs. 495 passive or ligament

Figure 5: Hip adduction (+)/abduction(-) (AA) and internal(+)/external(-) rotation (IER) - Kinematics, moment and power, net vs. passive or ligament

Figure 6: Knee extension(+)/flexion(-) (FE) - Kinematics, moment and power, net vs. 500 passive or ligament

Figure 7: Ankle flexion (+) / extension(-) (FE) - Kinematics, moment and power, net vs. passive or ligament 
505 Table Caption:

Table 1: Parameters of passive joint model $P^{1}$ following ISB standards for the joint axes

Table 2: Parameters of passive joint model $P^{2}$ following ISB standards for the joint axes

Table 3: Parameters of ligament model $L^{1}$ following ISB standards for the joint axes

510 Table 4: Parameters of ligament model $L^{2}$ following ISB standards for the joint axes

Table 5: Parameters of ligament model $L^{3}$ following ISB standards for the joint axes

Table 6: Contribution of the passive and ligament actions to the net joint moments and powers at the three joints of the lower limb. The contributions obtained by Whittington et al. (2008) [2] are given for comparison. 

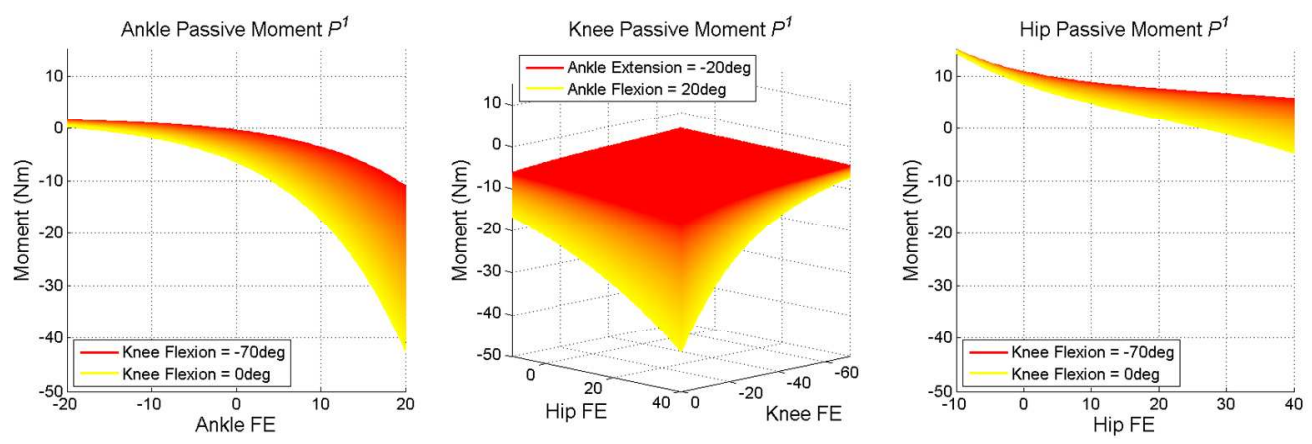

Figure 1. Passive joint moments of model L1 in function of joint angles on typical gait joint range of motion, the colour variation from red to yellow indicates the angle variation between the extreme values defined in the legend of the graph.

$356 \times 116 \mathrm{~mm}(120 \times 120 \mathrm{DPI})$ 

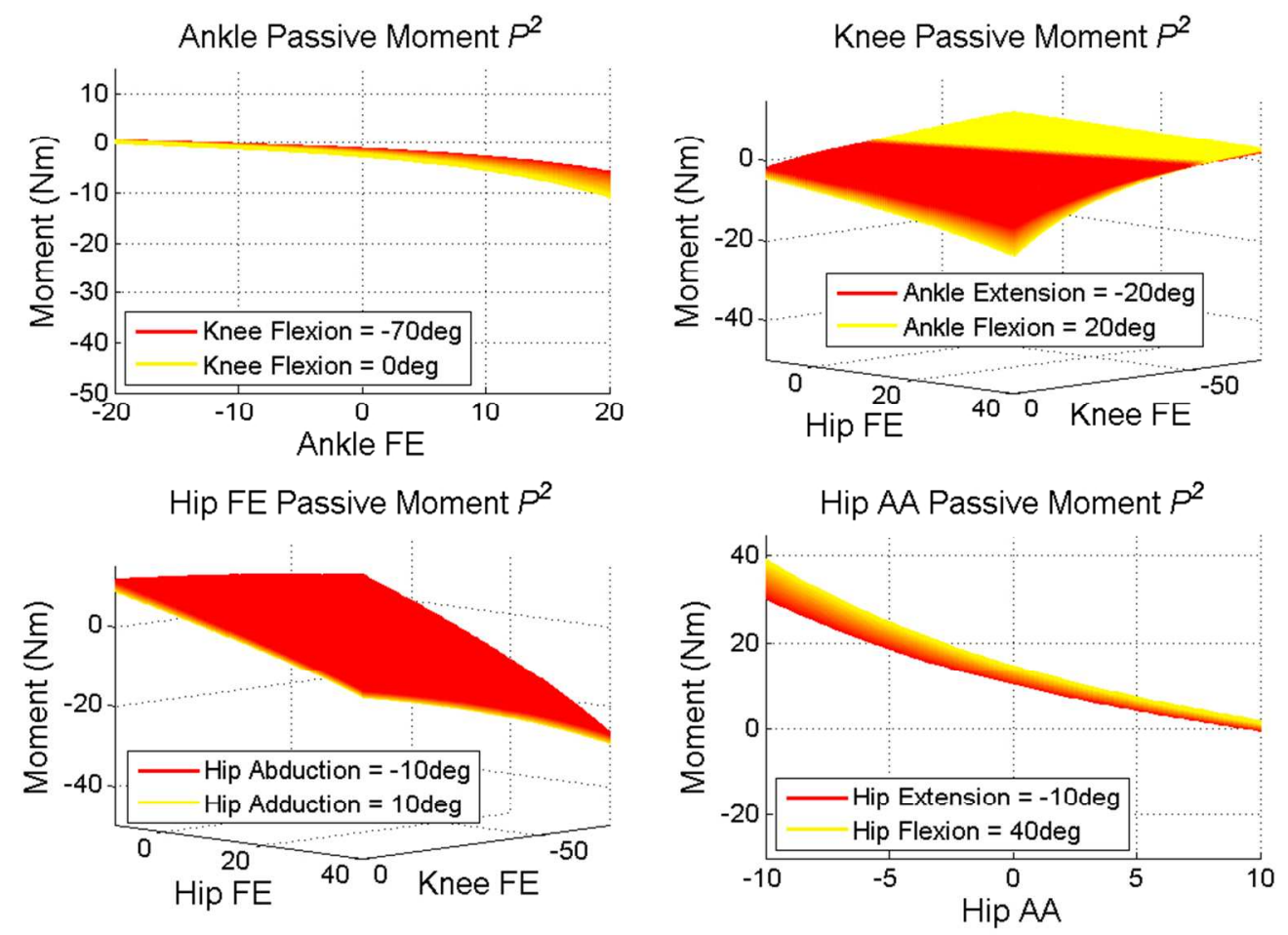

Figure 2. Passive joint moments of model $\mathrm{L} 2$ in function of joint angles on typical gait joint range of motion, the colour variation from red to yellow indicates the angle variation between the extreme values defined in the legend of the graph.

$218 \times 159 \mathrm{~mm}(120 \times 120 \mathrm{DPI})$ 

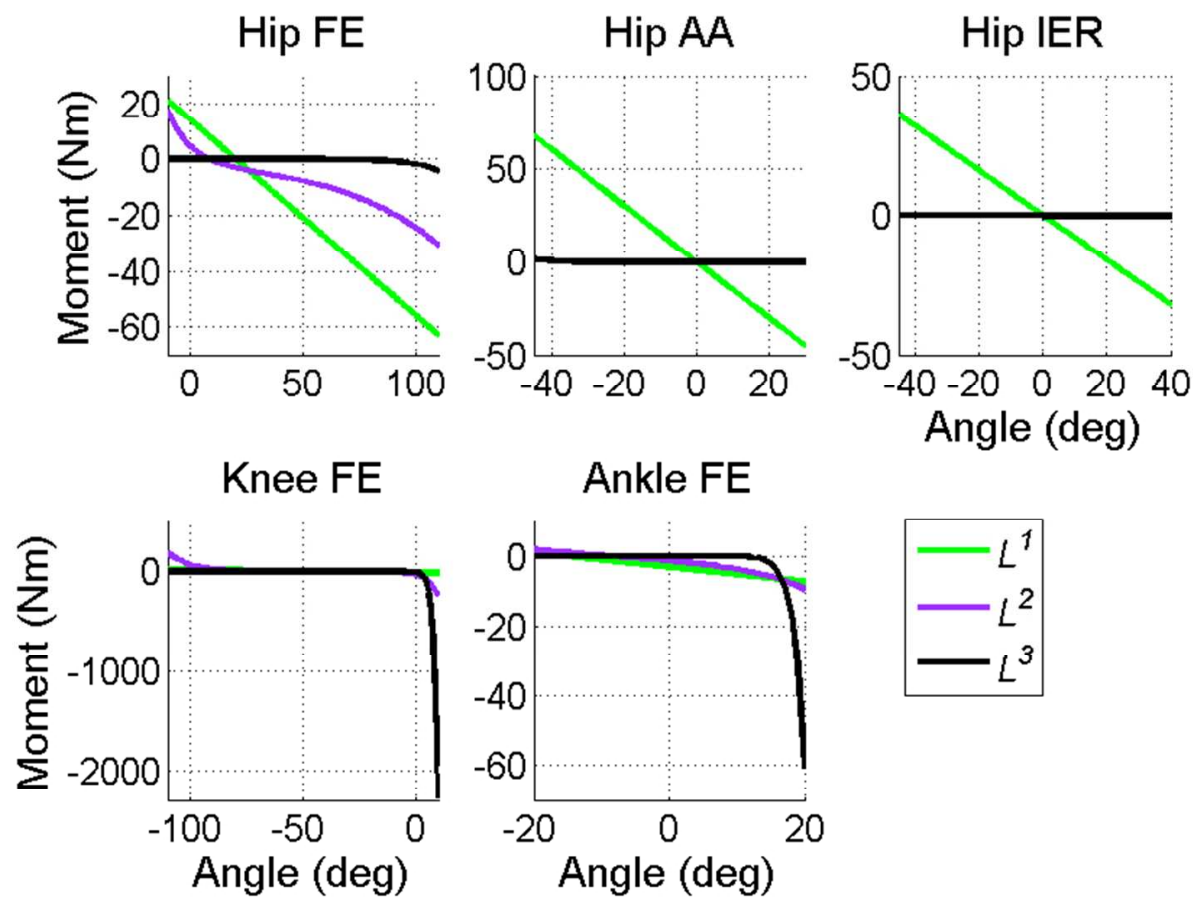

Figure 3. Ligaments moments of models $L 1, L 2$ and $L 3$ in function of joint flexion $197 \times 142 \mathrm{~mm}(116 \times 116 \mathrm{DPI})$ 

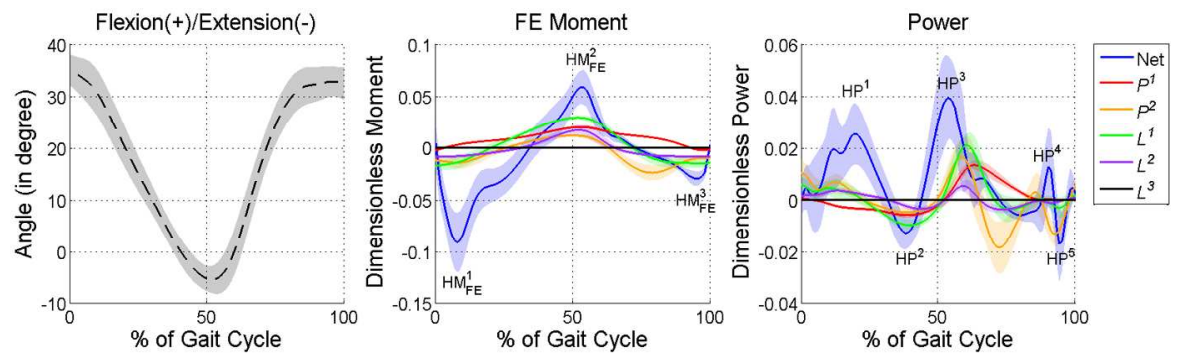

Figure 4. Hip flexion(+)/extension(-) (FE) - Kinematics, moment and power, net vs. passive or ligament $417 \times 110 \mathrm{~mm}$ (116 x 116 DPI) 

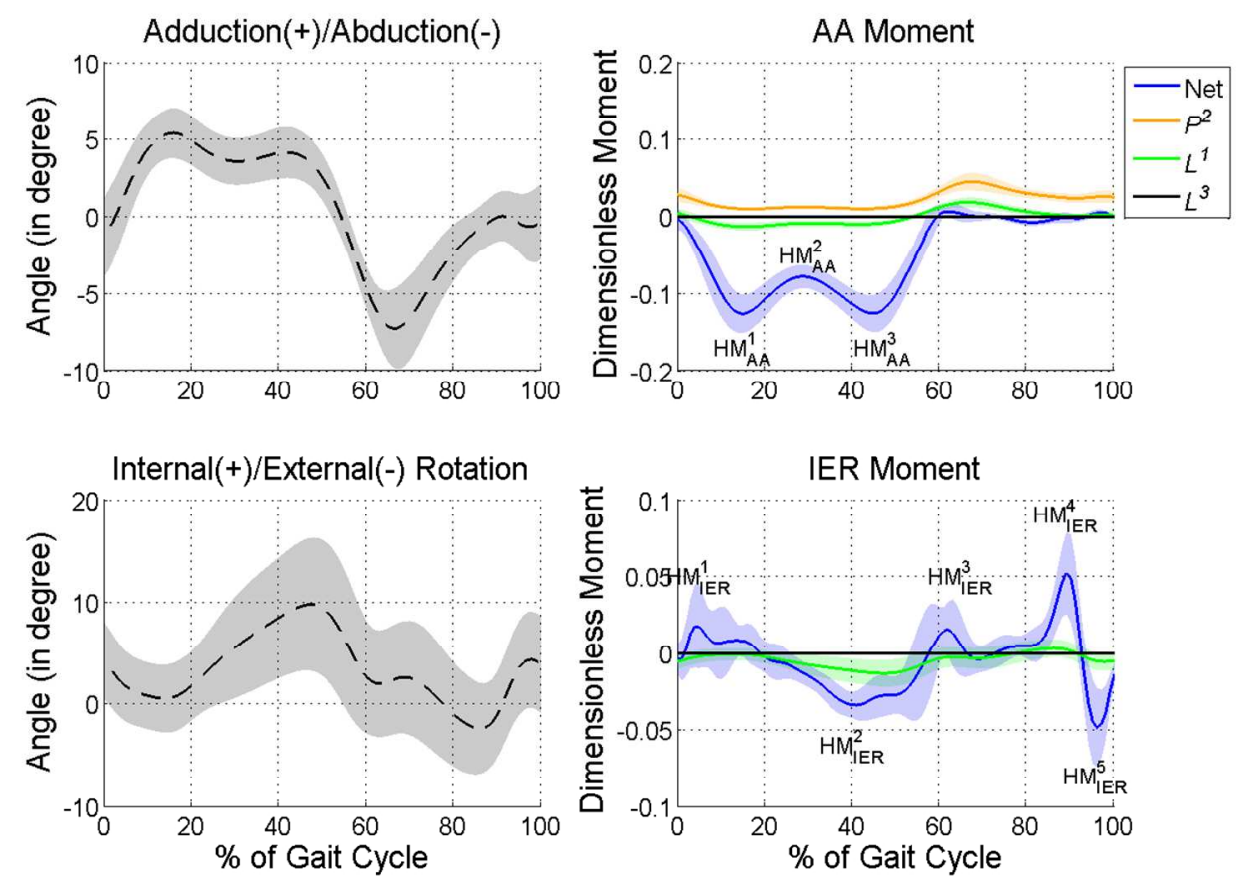

Figure 5. Hip adduction (+)/abduction(-) (AA) and internal(+)/external(-) rotation (IER) - Kinematics, moment and power, net vs. passive or ligament 

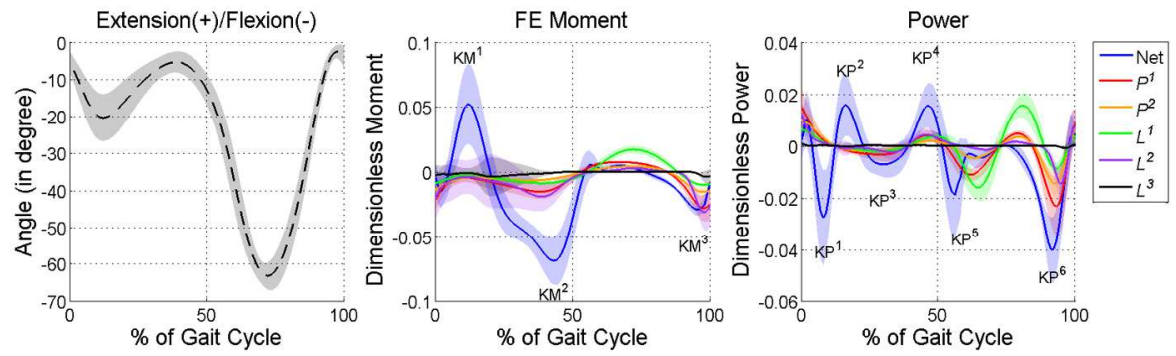

Figure 6. Knee extension(+)/flexion(-) (FE) - Kinematics, moment and power, net vs. passive or ligament $417 \times 110 \mathrm{~mm}(116 \times 116 \mathrm{DPI})$ 

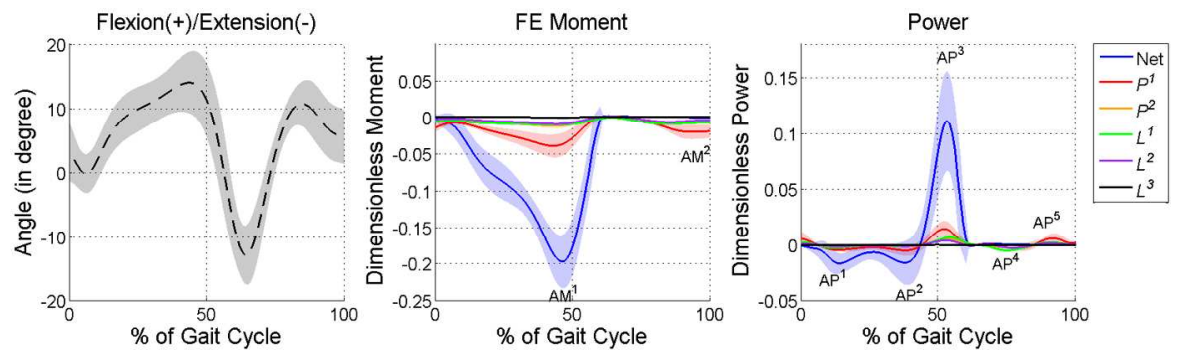

Figure 7. Ankle flexion (+) / extension(-) (FE) - Kinematics, moment and power, net vs. passive or ligament $418 \times 110 \mathrm{~mm}(116 \times 116$ DPI $)$ 


\section{Table 1}

\begin{tabular}{lccc}
\hline & Hip & Knee & Ankle \\
\cline { 2 - 4 } & FE & FE & FE \\
\hline$\alpha_{i}$ & 1.4655 & -3.9710 & -7.9763 \\
$\beta_{i}$ & 1.3403 & 1.8000 & 2.1016 \\
$h_{i}^{1}\left(\mathrm{deg}^{-1}\right)$ & -0.0750 & -0.0128 & 0 \\
$h_{i}^{2}\left(\mathrm{deg}^{-1}\right)$ & 0.0305 & 0.0217 & 0 \\
$k_{i}^{1}\left(\mathrm{deg}^{-1}\right)$ & 0.0034 & -0.0495 & -0.0008 \\
$k_{i}^{2}\left(\mathrm{deg}^{-1}\right)$ & 0.0226 & 0.0352 & 0.0176 \\
$a_{i}^{1}\left(\mathrm{deg}^{-1}\right)$ & 0 & 0.0004 & -0.1949 \\
$a_{i}^{2}\left(\mathrm{deg}^{-1}\right)$ & 0 & 0.0460 & 0.0843 \\
$C_{i}(\mathrm{~N} . \mathrm{m})$ & 8.072 & 4.820 & 1.792 \\
\hline
\end{tabular}


Table 2

\begin{tabular}{cccccc}
\hline & Hip & & Knee & Ankle & \\
\cline { 2 - 6 } & FE & AA & FE & FE & IE \\
\hline$a_{1}$ & -13 & 16 & -6.1 & -2.8 & 0.65 \\
$a_{2}$ & 0.87 & -4.5 & 2.3 & 3.9 & -2.8 \\
$a_{3}$ & 0.45 & - & -1.1 & 0.49 & 0.89 \\
$a_{4}$ & -0.051 & 0.27 & 0.82 & 0.22 & - \\
$a_{5}$ & 6.3 & -4.8 & 2 & 0.2 & -0.63 \\
$a_{6}$ & -1.3 & 2.3 & -0.5 & -4.1 & 3 \\
$a_{7}$ & -0.11 & - & 0.15 & 0.14 & 0.019 \\
$a_{8}$ & 0.65 & 0.021 & 0.47 & 1.5 & - \\
$\theta_{d}$ & Knee FE & Hip FE & Ankle FE & Ankle IE & - \\
$\theta_{p}$ & Hip AA & - & Hip FE & Knee FE & Ankle FE \\
\hline
\end{tabular}


Table 3

\begin{tabular}{cccccc}
\hline & Hip & & & Knee & Ankle \\
\cline { 2 - 6 } & FE & AA & IER & FE & FE \\
\hline$k_{i}\left(\mathrm{~N} \cdot \mathrm{m} /{ }^{\circ}\right)$ & 0.7 & 1.5 & 0.8 & 0.27 & 0.21 \\
$\theta_{i}^{0}\left({ }^{\circ}\right)$ & 20 & 0 & 0 & -25 & -15 \\
\hline
\end{tabular}


Table 4

\begin{tabular}{lccc}
\hline & Hip & Knee & Ankle \\
\cline { 2 - 4 } & FE & FE & FE \\
\hline$a\left(\mathrm{rad}^{-1}\right)$ & 5.8 & 5.9 & 5.0 \\
$b\left(\mathrm{rad}^{-1}\right)$ & 1.3 & 11.8 & 5.0 \\
$\alpha(\mathrm{rad})$ & 0.174 & -1.22 & -0.34 \\
$\beta(\mathrm{rad})$ & 0.95 & -0.09 & 0.34 \\
$c(\mathrm{~N} . \mathrm{m})$ & 2.6 & 3.1 & 2.0 \\
$d(\mathrm{~N} . \mathrm{m})$ & 8.7 & 10.5 & 5.0 \\
\hline
\end{tabular}


Table 5

\begin{tabular}{lccccc}
\hline & Hip & & & Knee & Ankle \\
\cline { 2 - 6 } & FE & AA & IER & FE & FE \\
\hline$a\left(\mathrm{rad}^{-1}\right)$ & 5.05 & 14.94 & 14.94 & 33.94 & 38.11 \\
$b\left(\mathrm{rad}^{-1}\right)$ & -21.88 & -14.94 & -14.94 & -11.33 & -42.12 \\
$\alpha(\mathrm{rad})$ & 1.81 & 0.5 & 0.92 & 0.0 & 0.26 \\
$\beta(\mathrm{rad})$ & -0.47 & -0.5 & -0.92 & -2.4 & -0.52 \\
$c(\mathrm{~N} . \mathrm{m})$ & -2.44 & -0.03 & -0.03 & -6.09 & -2.03 \\
$d(\mathrm{~N} . \mathrm{m})$ & 1.51 & 0.03 & 0.03 & 11.03 & 0.18 \\
\hline
\end{tabular}




\section{Table 6}

\begin{tabular}{|c|c|c|c|c|c|c|c|c|c|}
\hline Joint & & Peak & $P^{1}$ & $P^{2}$ & $L^{1}$ & $L^{2}$ & $L^{3}$ & {$[2]$} & Gait cycle phase \\
\hline \multirow[t]{5}{*}{ Hip } & \multirow[t]{3}{*}{ Moment } & $\begin{array}{l}\mathrm{HM}_{\mathrm{FE}}{ }^{1} \\
\mathrm{HM}_{\mathrm{FE}}{ }^{2} \\
\mathrm{HM}_{\mathrm{FE}}{ }^{2}\end{array}$ & $\begin{array}{r}0 \% \\
34 \% \\
0 \%\end{array}$ & $\begin{array}{l}16 \% \\
20 \% \\
80 \%\end{array}$ & $\begin{array}{l}14 \% \\
50 \% \\
48 \%\end{array}$ & $\begin{array}{r}9 \% \\
27 \% \\
27 \%\end{array}$ & $\begin{array}{l}0 \% \\
0 \% \\
0 \%\end{array}$ & $\begin{array}{r}0 \% \\
34 \% \\
0 \%\end{array}$ & $\begin{array}{l}\text { Double support } \\
\text { Toe-off } \\
\text { Late swing }\end{array}$ \\
\hline & & $\begin{array}{l}\mathrm{HM}_{\mathrm{AA}}{ }^{1} \\
\mathrm{HM}_{\mathrm{AA}}\end{array}$ & & $\begin{array}{l}-7 \% \\
-8 \%\end{array}$ & $\begin{array}{r}10 \% \\
7 \%\end{array}$ & & $\begin{array}{l}0 \% \\
0 \%\end{array}$ & & $\begin{array}{r}\text { Opposite toe off } \\
\text { Opposite foot contact }\end{array}$ \\
\hline & & $\begin{array}{l}\mathrm{HM}_{\text {IER }}^{2} \\
\mathrm{HM}_{5}{ }_{\text {IER }}\end{array}$ & & & $\begin{array}{l}20 \% \\
11 \%\end{array}$ & & $\begin{array}{l}0 \% \\
0 \%\end{array}$ & & $\begin{array}{l}\text { Single Support } \\
\text { Late swing }\end{array}$ \\
\hline & \multirow[t]{2}{*}{ Power } & $\mathrm{HP}^{2}$ & $47 \%$ & $37 \%$ & $78 \%$ & $20 \%$ & $0 \%$ & $34 \%$ & Single support \\
\hline & & $\mathrm{HP}^{3}$ & $37 \%$ & $42 \%$ & $79 \%$ & & $13 \%$ & $0 \%$ & $53 \% \quad$ Push off \\
\hline \multirow[t]{2}{*}{ Knee } & Moment & $\begin{array}{l}\mathrm{KM}^{2} \\
\mathrm{KM}^{3}\end{array}$ & $\begin{array}{l}23 \% \\
98 \%\end{array}$ & $\begin{array}{l}10 \% \\
53 \%\end{array}$ & $\begin{array}{l}13 \% \\
35 \%\end{array}$ & $\begin{array}{r}28 \% \\
115 \%\end{array}$ & $\begin{array}{l}0 \% \\
0 \%\end{array}$ & $\begin{array}{l}-4 \% \\
46 \%\end{array}$ & $\begin{array}{l}\text { Single support } \\
\text { Late swing }\end{array}$ \\
\hline & Power & $\begin{array}{l}\mathrm{KP}^{3} \\
\mathrm{KP}^{4} \\
\mathrm{KP}^{5} \\
\mathrm{KP}^{6}\end{array}$ & $\begin{array}{l}47 \% \\
25 \% \\
65 \% \\
62 \%\end{array}$ & $\begin{array}{l}29 \% \\
12 \% \\
25 \% \\
36 \%\end{array}$ & $\begin{array}{l}36 \% \\
26 \% \\
88 \% \\
24 \%\end{array}$ & $\begin{array}{l}28 \% \\
22 \% \\
42 \%\end{array}$ & $\begin{array}{c}0 \% \\
0 \% \\
7 \% \quad 0 \\
0 \%\end{array}$ & $\begin{array}{c}\% \quad 13 \\
27 \%\end{array}$ & $\begin{array}{l}\text { Single support } \\
\text { Push off } \\
3 \% \quad \text { Toe-off } \\
\text { Late swing }\end{array}$ \\
\hline \multirow[t]{2}{*}{ Ankle } & Moment & $\mathrm{AM}^{1}$ & $20 \%$ & $6 \%$ & $5 \%$ & $3 \%$ & $0 \%$ & $19 \%$ & Push-off \\
\hline & Power & $\begin{array}{l}\mathrm{AP}^{1} \\
\mathrm{AP}^{2} \\
\mathrm{AP}^{3}\end{array}$ & $\begin{array}{l}27 \% \\
30 \% \\
13 \%\end{array}$ & $\begin{array}{r}12 \% \\
9 \% \\
5 \%\end{array}$ & $\begin{array}{r}16 \% \\
8 \% \\
6 \%\end{array}$ & $7 \%$ & $\begin{array}{c}8 \% \\
0 \%\end{array}$ & $\begin{array}{l}0 \% \quad 5 \\
27 \% \\
4 \%\end{array}$ & $\begin{array}{l}55 \% \text { Opposite toe off } \\
\text { Opposite foot contact } \\
3 \% \quad 11 \% \quad \text { Push off }\end{array}$ \\
\hline
\end{tabular}

